\title{
Effectiveness of craniosacral therapy in cervicogenic headache
}

\begin{abstract}
Background: Headache is common phenomenon experienced by everyone at some point in their lives. Hence it's not surprising that the global estimate of headache sufferers is around $46 \%$ and prevalence of Cervicogenic headache is $2.5-4.1 \%$ among all headache types. This prevalence might appear to be relatively low as compared to other headache types like migraine or tension type headache but the associated disability is alarming and high. Craniosacral therapy is a light touch modality which is fairly safe, non-invasive and non-pharmacological mode of treatment which can cure a wide array of musculoskeletal and neurological conditions including headaches but which has limited evidence of effectiveness in literature especially in Cervicogenic Headache. Hence this study aimed at assessing the effectiveness of craniosacral therapy as a treatment modality in the management in Cervicogenic headache.
\end{abstract}

Methods: This study hypothesized that Craniosacral Therapy will demonstrate better improvement in all sub-sections of the Headache Impact Test (HIT-6) which measures the quality of life and also demonstrate reduced frequency and duration of Cervicogenic headache as measured by a self-maintained headache diary. Selection criteria for inclusion in the study were subjects of both gender, age 18 and above and meeting the Cervicogenic Headache International Study Group diagnostic criteria for Cervicogenic Headache. All subjects were treated 3 times a week on alternate days for 3 weeks.

Results: 94 individuals were screened for eligibility of which 49 met the selection criteria for the study. Headache-related disability was present $3.4 \pm 4.1$ days during the 3 week period. The average of the HIT- 6 score pre- treatment was $67.6 \pm 7.8$ points and post- treatment was $42.7 \pm 3.6$. The Correlation analysis of the frequency of headache attacks and duration of disability according to the headache diary significantly correlated with the severity of headache-related disability at each attack.

Conclusion: Craniosacral therapy is an effective treatment strategy for patients of Cervicogenic Headache as measured on HIT-6 during a 3week treatment program.

Keywords: cervicogenic headache, craniosacral therapy, HIT-6, headache diary
Volume 2 Issue 4 - 2017

\author{
Keerthi Rao,' Subhash Khatri² \\ 'Associate Professor, APJAK College of Physiotherapy, India \\ 2Principal, APJAK College of Physiotherapy, India
}

Correspondence: Keerthi Rao, Associate Professor,APJAK College of Physiotherapy, India, Tel +9190499|3244, Email keerthimpt@gmail.com

Received: October 28, 2016 | Published: December 14, 2017

\section{Abbreviations: CGH, cervicogenic headache; CST, craniosacral therapy \\ Introduction}

Headache is common phenomenon experienced by everyone at some point in their lives. Hence it's not surprising that the global estimate of headache sufferers is around $46 \%$ and prevalence of Cervicogenic headache is $2.5-4.1 \%$ among all headache types. ${ }^{1,2}$ This prevalence might appear to be relatively low as compared to other headache types like migraine or tension type headache but the associated disability is alarming and high. Cervicogenic Headache (CGH) arises mainly from dysfunction in the first three upper cervical segments. ${ }^{3}$ The probable pathway by which pain initiating in the neck can be referred as a headache is the trigeminocervical nucleus which descends in the spinal cord to the area of $\mathrm{C} 3$ or sometimes $\mathrm{C} 4$. These structures are further in anatomical continuity with the dorsal gray columns of the same spinal segments. ${ }^{4}$ Therefore, input from sensory afferents primarily from any of the upper three cervical nerve roots can be mistaken to be perceived as pain in the head ${ }^{3}$ through a process called as convergence. Although the pathophysiology is not totally clear there are definite articular, muscular and neural mechanisms which are at play. ${ }^{5}$ The average age of onset has been marked as 33-43years and the mean of the duration of presence of symptoms at 7-17 years. ${ }^{1,2}$ The chronicity seems to develop through increase in frequency of headache which are short lasting and not continuous or unrelenting.

Various management strategies start with pharmacological medications but they have little role $^{6}$ in the long course of such headaches so other strategies like manual and manipulative therapy, ${ }^{7-12}$ Low Level Laser Therapy, ${ }^{13-15}$ recommendations for sleep, exercise, stress reduction through behavioral interventions are found to be effective for treatment of CGH. The effectiveness of nonpharmacological interventions for the treatment of CGH is warranted to reduce the side effects of medications and to provide comfort of intervention.

Craniosacral therapy (CST) is a light touch modality which is fairly safe, non- invasive and non-pharmacological approach which is applied as a gentle manual force to address somatic dysfunctions of the head and the remainder of the body. This treatment is aimed at mobilizing the cranial sutures which are restricted leading to a loss of 
normal physiologic motion. Restrictions in the Craniosacral system are manually identified which include the bones, membranes and cerebrospinal fluid (CSF) that surround the brain and spinal cord ${ }^{16}$ using soft, gentle hands-on techniques to both normalize the CST fluid rhythm and correct restrictions in peri-spinal tissues and fascia for the treatment and prevention of $\mathrm{CGH}$ for which there is limited evidence of safety and efficacy. Manual palpation and manipulation of this system theoretically affects sensory, motor, cognitive and emotional processes in the nervous system. ${ }^{16-18}$ Several studies report, benefit of CST in various types of headache but these studies have not been done specifically on CGH and also lack proper documentation on how CST is beneficial with respect to the quality-of-life. Hence this study aims at evaluating the effectiveness of CST in CGH as measured by HIT-6.

\section{Material and methods}

Selection criteria for inclusion were: subjects of both gender, age 18 years and above, meeting the Cervicogenic Headache International Study Group diagnostic criteria ${ }^{19}$ for CGH. Exclusion criteria were sudden onset of a new severe headache, a worsening pattern of a preexisting headache in the absence of obvious predisposing factors, headache associated with fever, neck stiffness, skin rash and with a history of cancer, (document the full terms of the medical expression of HIV firstly) HIV, or other systemic illness, headache associated with focal neurologic signs other than typical aura, moderate or severe headache triggered by cough, exertion, new onset of a headache during or following pregnancy, ${ }^{20}$ cranial tumors, meningitis, giant cell arteritis, sub-arachnoid hemorrhage and carotid artery or vertebral artery dissection. ${ }^{21}$

The subjects were referred to Orthopedic Physiotherapy Department after thorough medical assessment from the Medicine, Surgery and Orthopedic Departments. Those meeting the selection criteria were informed about the study procedure and a written consent was obtained from them. Excluded subjects were referred back to their physicians for further medical management. Enrolled subjects were asked to avoid changes in medications for headache during the course of the study. All subjects were assessed for Headache Impact Test- 6 and asked to maintain a headache diary for 3 consecutive weeks.

Subjects received three CST sessions on alternate days per week for 3 (consecutive!?) weeks amounting to a total of 9 sessions. Since there were no published studies reporting evidence-based treatment schedules for CST, the number of treatments was based on data from a pilot study (document short description about the applied pilot study) performed prior to this study and opinions of qualified, experienced therapists in the field (refer to their researches or cases study performed).

The subject lies supine on a couch and is completely clothed in comfortable attire but accessories like belts, shoes, jewellery, hair bands and watches are removed while the therapist evaluates the craniosacral system. The CST protocol for each subject follows the 10 -step protocol Version 1 as suggested by Upledger Institute shown in Table 1.
Table I 10 step CST protocol version I for each subject

\begin{tabular}{ll}
\hline S. No & Contents \\
\hline 1. & Still point \\
2. & Diaphragm releases \\
3. & Frontal lift \\
4. & Parietal lift \\
5. & Spheno basilar compression- decompression \\
6. & Temporal bone techniques \\
7. & Temporal decompression \\
8. & Tempero mandibular compression decompression \\
9. & Dural tube evaluation \\
10. & CV-4/ Still point \\
\hline
\end{tabular}

\section{Results and discussion}

The HIT-6 consists of six items: pain, social functioning, and role functioning, vitality, cognitive functioning and psychological distress. ${ }^{22}$ The patient answers each of the six related questions using one of the following five responses: "never", "rarely", "sometimes", "very often", or "always". These responses are summed to produce a total HIT-6 score that ranges from 36 to 78 , where a higher score indicates a greater impact of headache on the daily life of the respondent. $^{23-25}$

The subjects were requested to maintain a headache diary from the first visit for 3 weeks till the study intervention was carried out, along with sufficient information about the required contents. The subjects were further instructed to complete the diary every night and specifically on days on which they experienced a headache. Each diary was maintained for 3-week duration and it contained questions on headache characteristics and other associated symptoms during the attack. The headache pain intensity was quantified using a visual analog scale measuring from 0 to 10 where 0 meant no pain and 10 meant worse imaginable pain.

SPSS statistical software (version 10.0) was used for all the analyses. Correlation analysis was used to verify the relationships of the HIT-6 score with headache features and disability from the headache diary. A probability value of $<0.01$ was considered statistically significant. During the diary recording period of 3 weeks, 49 subjects of the study contributed 121 diary entries of headache. The demographic data shows that females comprised $71 \%$ of the participants. Headache-related disability was present $3.4 \pm 4.1$ days during the 3 week period. The average of the HIT- 6 score pretreatment was $67.6 \pm 7.8$ points and post- treatment was $42.7 \pm 3.6$. The Correlation analysis of the frequency of headache attacks and duration of disability according to the headache diary significantly correlated with the severity of headache-related disability at each attack.

$\mathrm{CGH}$ is fairly debilitating to the sufferer during each attack and medications give temporary relief and have numerous side effects in the long run. In this study, we evaluated the effectiveness of CST and 
found it to be ineffective in treating CGH. The probable causes can be that the craniosacral system operates like a semi-closed hydraulic system. There is a rhythmic rise and fall of cerebrospinal fluid volume and pressure within the boundaries formed by the dural mater. According to research performed at Michigan State University ${ }^{26-32}$ the cranial bones with their dural linings are in continual, minute motion to accommodate the constant fluid pressure changes within the membrane compartment. The cerebrospinal fluid within the craniosacral system acts as a shock absorber for the brain. In addition to delivering nutrients to the nerves, brain and spinal cord tissue, the fluid washes away waste products emanating from metabolic processes and thus reduces pain.

Research has shown that the meningeal membranes and the perivascular fascia are the only pain-sensitive tissues in the brain. Therefore, any abnormal meningeal tension can cause pain, as can any pressure on blood vessels. Abnormal meningeal tension or aberrant pressures on the brain stem from surrounding fascia can also potentially cause postsynaptic sensory neurons to relay their messages to higher brain centers. This relates to another theory that pain receptors in upper cervical segments actually cause the Cervicogenic headache and Craniosacral Therapy helps prevent and abort headaches primarily by releasing tensions throughout the meninges. By removing restrictions from meningeal and cranial bone structures, pressure is taken off the nervous system and the entire craniosacral system can open up. This also allows fluid to drain so back pressure does not build up. The dural covers the inside of the cranial bones and surrounds the foramen magnum. It exits the cranium and attaches to $\mathrm{C} 2$ and $\mathrm{C} 3$, continuing down to where it attaches at S2 and the coccyx. Thus, it forms the dural tube that surrounds the spinal cord.

When nerve roots refer increased levels of impulse activity into the spinal cord from their peripheral domains, a facilitated condition of the related spinal cord segments occurs. A condition of hyperactivity in the facilitated spinal cord segments sends out impulses to the related dural tube and dural sleeves. This causes a tightening and loss of mobility of the dural tube related to the facilitated segments with increased nerve pressure from a contracted dural tube sleeve resulting in continual neuronal firing. Also, the nerves in the area go to the intervertebral muscle, causing them to contract and cause fixation and subluxation. If a peripheral restriction is released but the dural tube restriction and facilitated spinal cord segments are not, the peripheral problem usually reoccurs. So a peripheral problem can translate through the facilitated segments up into the cranium and cause the meninges to contract in the same way an intracranial meningeal problem can translate down the dural tube and cause facilitation. Either one can result in a Cervicogenic headache. Craniosacral Therapy has effectively helped release such dural tube restrictions to normalize the activity of facilitated spinal cord segments.

\section{Conclusion}

This study revealed that craniosacral therapy is an effective treatment strategy for patients of Cervicogenic Headache as measured by HIT-6.

\section{Acknowledgements}

None.

\section{Conflict of interest}

The author declares no conflict of interest.

\section{References}

1. Haldeman S, Dagenais S. Cervicogenic headaches: A critical review. Spine J. 2001;1(1):31-46.

2. Sjaastad O, Bakketeig LS. Prevalence of Cervicogenic headache: Vaga study of headache epidemiology. Acta Neurol Scand. 2008;117(3):170 183.

3. Bogduk N. Headache and the neck. In: Goadsby P, et al. editors. Headache. Australia: Butterworth-Heinemann; 1997.

4. Edmeads J. Disorders of the neck: Cervicogenic headache. In: Silberstein SD, et al. editors. Wolff's Headache and Other Head Pain. UK: Oxford University Press; 2001.

5. Brandes JL, Saper JR, Diamond M, et al. Topiramate for migraine prevention: a randomized controlled trial. JAMA. 2004;291(8):965-973.

6. Bogduk N, Govind J. Cervicogenic headache: an assessment of the evidence on clinical diagnosis, invasive tests and treatment. Lancet Neurol. 2009;8(10):959-968.

7. Haas M, Groupp E, Aickin M, et al. Dose response for chiropractic care of chronic cervicogenic headache and associated neck pain: randomized pilot study. J Manipulative Physiol Ther. 2004;27(9):547-553.

8. Haas M, Spegman A, Peterson D, et al. Dose response and efficacy of spinal manipulation for chronic cervicogenic headache: a pilot randomized controlled trial. Spine J. 2010;10(2):117-128.

9. Jull G, Trott P, Potter H, et al. A randomized controlled trial of exercise and manipulative therapy for cervicogenic headache. Spine. 2002;27(17):18351843 .

10. Nilsson N. A randomized controlled trial of the effect of spinal manipulation in the treatment of cervicogenic headache. J Manipulative Physiol Ther. 1995;18(7):435-440.

11. Schoensee SK, Jensen G, Nicholson G, et al. The effect of mobilization on cervical headaches. J Orthop Sports Phys Ther. 1995;21(4):184-196.

12. Sylva DJ, Miller J, Gross A, et al. Manual therapy with or without physical medicine modalities for neck pain: a systematic review. Man Ther. 2010;15(5):415-433.

13. Chow RT, Johnson MI, Martins LRA, et al. Efficacy of low-level laser therapy in the management of neck pain: a systematic review and metaanalysis of randomised placebo or active-treatment controlled trials. Lancet. 2009;374(9705):1897-1908.

14. Leaver AM, Refshauge KM, Maher CG, et al. Conservative interventions provide short-term relief for non-specific neck pain: a systematic review. $J$ Physiother. 2010;56(2):73-85.

15. Ebneshahidi NS, Heshmatipour M, Moghaddami A, et al. The effects of laser acupuncture on chronic tension headache a randomised controlled trial. Acupunct Med. 2005;23(1):13-18.

16. Upledger JE. CranioSacral Therapy. In: Novey DW, editors. Clin-ician's complete reference to complementary and alternative medicine. USA: St Louis Mosby; 2000. p. 381-392.

17. Downey PA, Barbano T, Wadhwa KR, et al. Craniosacral therapy: the effects of cranial manipulation on intracranial pressure and cranial bone movement. J Orthop Sports Phys Ther. 2006;36(11):845-853.

18. Ferguson A. A review of the physiology of cranial osteopathy. Journal of Osteopathic Medicine. 2003;6(2):74-84.

19. Sjaastad O, Fredriksen TA, Pfaffenrath V. Cervicogenic headache: diagnostic criteria. The cervicogenic headache international study group. Headache. 1998;38:442-445.

20. Bigal ME, Lipton RB. The differential diagnosis of chronic daily headaches: 
An algorithm based approach. J Headache Pain. 2007;8(5):263-272.

21. Landtblom AM, Fridriksson S, Boivie J, et al. Sudden onset headache: A prospective study of features, incidence and causes. Cephalalgia. 2002;22(5):354-360.

22. Bayliss MS, Dewey JE, Dunlap I, et al. A study of the feasibility of Internet administration of a computerized health survey: the headache impact test (HIT). Qual Life Res. 2003;12(8):953-961.

23. Kosinski M, Bayliss MS, Bjorner JB, et al. A six item short form survey for measuring headache impact: the HIT-6. Qual Life Res. 2003;12(8):963974.

24. Ware JE, Bjorner JB, Kosinski M. Practical implications of item response theory and computerized adaptive testing: a brief summary of ongoing studies of widely used headache impact scales. Med Care. 2000;38(S9):II73-82.

25. Retzlaff EW. Nerve fibers and endings in cranial sutures research report JAOA. 1978;77:474-475.
26. Retzlaff EW. Possible functional significance of cranial bone sutures report. USA: 88th Session American Association of Anatomists; 1975.

27. Retzlaff EW. Structure of cranial bone sutures research report. JAOA 1976;75:607-608.

28. Retzlaff EW. Sutural collagenous and their innvervation in saimiri sciurus. Anatomical Record. 1977;187:692.

29. Retzlaff EW, Mitchell FL. The Cranium and its Sutures. Germany: Springer Verlag; 1987.

30. Upledger JE. The reproducibility of craniosacral examination findings: a statistical analysis. J Am Osteopath Assoc. 1977;76(12):890-899.

31. Upledger JE. The relationship of craniosacral examination findings in grade school children with developmental problems. J Am Osteopath Assoc. 1978;77(10):760-776.

32. Upledger JE, Karni Z. Mechano-electric patterns during craniosacral osteopathic diagnosis and treatment. $J$ Am Osteopath Assoc. 1979;78(11):782-791. 